\title{
Correction: Opportunities and Challenges for Digital Social Prescribing in Mental Health: Questionnaire Study
}

Shivani Patel ${ }^{1}$, MRCPsych; Gerry Craigen ${ }^{2,3}$, MD; Mariana Pinto da Costa ${ }^{1,4,5}$, MD; Becky Inkster ${ }^{6,7}$, DPhil

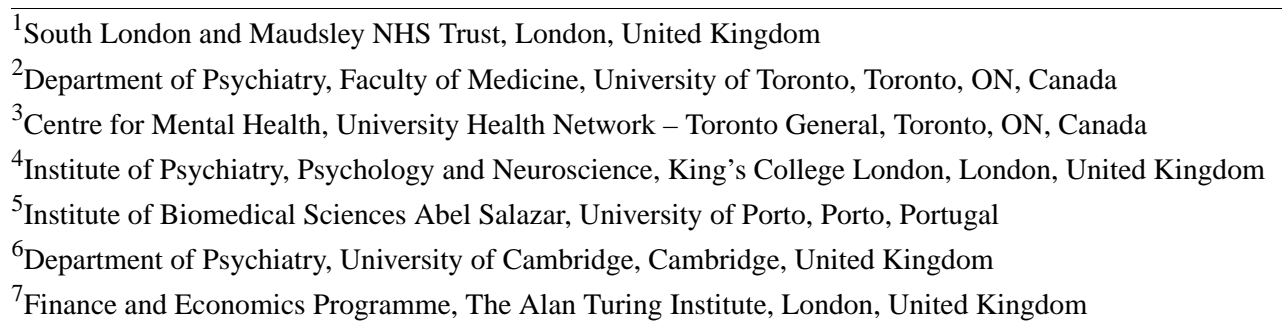

Corresponding Author:

Shivani Patel, MRCPsych

South London and Maudsley NHS Trust

Denmark Hill

London,

United Kingdom

Phone: 4432286000 ext 1234

Email: shivaninpatel183@gmail.com

\section{Related Article:}

Correction of: https://www.jmir.org/2021/3/e17438/

(J Med Internet Res 2021;23(3):e29041) doi: 10.2196/29041

In "Opportunities and Challenges for Digital Social Prescribing in Mental Health: Questionnaire Study" (J Med Internet Res 2021;23(3):e17438) the authors noted three errors.

In the originally published article, affiliation 1 was not applied to author Mariana Pinto da Costa, and the affiliation "Centre for Mental Health, University Health Network - Toronto General, Toronto, ON, Canada" was not added for author Gerry Craigen.

The author's affiliation section was originally published as follows:

Shivani Patel ${ }^{1}$, MRCPsych; Gerry Craigen ${ }^{2}, M D$; Mariana Pinto da Costa ${ }^{3,4}$, MD; Becky Inkster ${ }^{5,6}$, DPhil

${ }^{1}$ South London and Maudsley NHS Trust, London, United Kingdom

${ }^{2}$ Department of Psychiatry, Faculty of Medicine, University of Toronto, Toronto, ON, Canada

${ }^{3}$ Institute of Psychiatry, Psychology and Neuroscience, King's College London, London, United Kingdom

${ }^{4}$ Institute of Biomedical Sciences Abel Salazar, University of Porto, Porto, Portugal

${ }^{5}$ Department of Psychiatry, University of Cambridge, Cambridge, United Kingdom
${ }^{6}$ Finance and Economics Programme, The Alan Turing Institute, London, United Kingdom

The corrected author's affiliation section appears as follows:

Shivani Patel ${ }^{1}$, MRCPsych; Gerry Craigen ${ }^{2,3}, M D$; Mariana Pinto da Costa ${ }^{1,4,5}$, MD; Becky Inkster ${ }^{6,7}$, DPhil

${ }^{1}$ South London and Maudsley NHS Trust, London, United Kingdom

${ }^{2}$ Department of Psychiatry, Faculty of Medicine, University of Toronto, Toronto, ON, Canada

${ }^{3}$ Centre for Mental Health, University Health Network - Toronto General, Toronto, ON, Canada

${ }^{4}$ Institute of Psychiatry, Psychology and Neuroscience, King's College London, London, United Kingdom

${ }^{5}$ Institute of Biomedical Sciences Abel Salazar, University of Porto, Porto, Portugal

${ }^{6}$ Department of Psychiatry, University of Cambridge, Cambridge, United Kingdom

${ }^{7}$ Finance and Economics Programme, The Alan Turing Institute, London, United Kingdom

In the "Data Analysis" section, a correction has been made to clarify the author order referenced in the text. 
The following sentence appeared in the originally published manuscript:

The first author $(S P)$ coded all the material, and the second author (MP) reviewed all the data to ensure the consistency and credibility of the coding and grouping [18].

This sentence has been corrected to:

The first author $(S P)$ coded all the material, and the third author $(M P)$ reviewed all the data to ensure the consistency and credibility of the coding and grouping [18].

The correction will appear in the online version of the paper on the JMIR Publications website on March 29, 2021, together with the publication of this correction notice. Because this was made after submission to PubMed, PubMed Central, and other full-text repositories, the corrected article has also been resubmitted to those repositories.

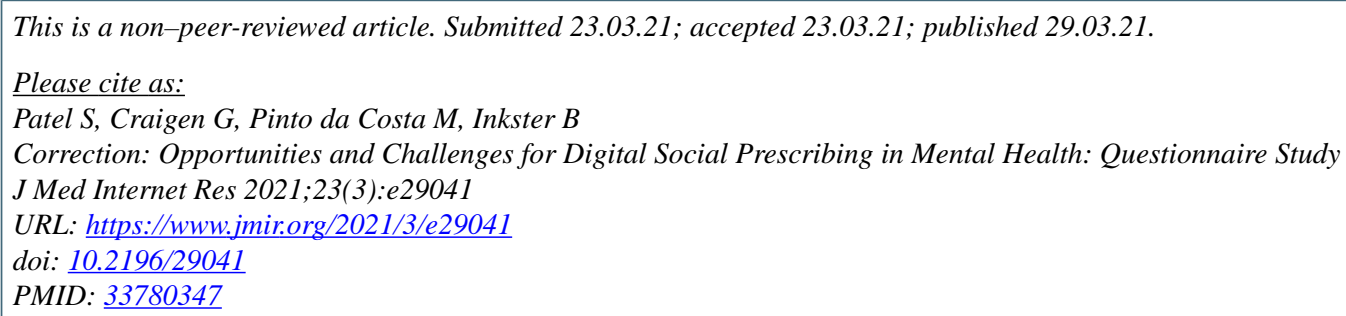

(C) Shivani Patel, Gerry Craigen, Mariana Pinto da Costa, Becky Inkster. Originally published in the Journal of Medical Internet Research (http://www.jmir.org), 29.03.2021. This is an open-access article distributed under the terms of the Creative Commons Attribution License (https://creativecommons.org/licenses/by/4.0/), which permits unrestricted use, distribution, and reproduction in any medium, provided the original work, first published in the Journal of Medical Internet Research, is properly cited. The complete bibliographic information, a link to the original publication on http://www.jmir.org/, as well as this copyright and license information must be included. 